\title{
Médiévales
}

Langues, Textes, Histoire

60 | printemps 2011

La fitna

\section{La richesse des Franciscains. Autour du débat sur les rapports entre économie et religion au Moyen Âge}

\section{Valentina Toneatto}

\section{(2) OpenEdition Journals \\ Édition électronique \\ URL : https://journals.openedition.org/medievales/6220 \\ DOI : $10.4000 /$ medievales. 6220 \\ ISSN : 1777-5892 \\ Éditeur}

Presses universitaires de Vincennes

\section{Édition imprimée}

Date de publication : 30 juin 2011

Pagination : 187-202

ISBN : 978-2-84292-273-3

ISSN : 0751-2708

Référence électronique

Valentina Toneatto, "La richesse des Franciscains. Autour du débat sur les rapports entre économie et religion au Moyen Âge », Médiévales [En ligne], 60 I printemps 2011, mis en ligne le 19 janvier 2012, consulté le 22 avril 2022. URL : http://journals.openedition.org/medievales/6220 ; DOI : https:// doi.org/10.4000/medievales.6220 
Valentina TONEATTO

\section{LA RICHESSE DES FRANCISCAINS. AUTOUR DU DÉBAT SUR LES RAPPORTS ENTRE ÉCONOMIE ET RELIGION AU MOYEN ÂGE}

La littérature historique sur les positions de l'Église vis-à-vis des échanges matériels et de la richesse rencontre souvent l'opinion commune selon laquelle la doctrine morale chrétienne aurait condamné la richesse matérielle et se serait opposée purement et simplement à l'enrichissement et au négoce. De la prédication patristique à la littérature théologique et à l'exégèse médiévale, les sources ecclésiastiques semblent sanctionner inlassablement le profit et la volonté d'acquérir les biens terrestres. Ainsi, lorsqu'on affronte le problème des rapports entre l'Église et le monde, on se heurte le plus souvent à l'idée, bien ancrée dans la pensée occidentale, d'une diversité structurelle opposant les champs du spirituel et de l'économique ${ }^{1}$, celui-ci étant caractérisé par son péché propre: l'avarice, la «racine de tous les maux», selon les mots de l'apôtre $(1$ Tim. 6,10$)$. Le domaine que nous qualifions d'économique - c'est-à-dire la sphère de la vie quotidienne ici-bas - semble qualitativement différent de la spiritualité chrétienne car il tend à

1. Les synthèses des manuels universitaires d'histoire du Moyen Âge donnent une idée de ces positions. Pour des références plus ponctuelles, échelonnées dans le temps, voir: E. TroeltsCH, Die Soziallehren der christlichen Kirchen und Gruppen, Tübingen, 1912 (trad. angl., The Social Teaching of the Christian Churches, Louisville, 1931; trad. it., Le dottrine sociali delle Chiese e dei gruppi cristiani, Florence, 1941) où il est question d'incompatibilité logique entre la morale chrétienne et le profit économique; plus récemment: L. MARTELLo éd., Sulla genesi del capitalismo, Rome, 1992; H.W. GoETZ, «Idéologie (et anti-idéologie) de la richesse au Haut Moyen Âge», dans J.-P. Devroey, L. Feller et R. Le JAn éd., Les Élites et la richesse au haut Moyen Âge, Actes du colloque international (Bruxelles, 13-15 mars 2008), Turnhout, 2010 (haut Moyen Âge, 10), p. $33-58$. 
la satisfaction des besoins matériels et individuels. Cette dichotomie reflète donc l'opposition couramment admise entre l'ici-bas et l'au-delà. Si l'on présuppose ainsi un conflit irréductible entre la morale chrétienne et le monde des affaires, le rapport du fidèle à la richesse ne peut pas être envisagé autrement qu'en termes de péché ou de renoncement au matériel en fonction du spirituel. En voyant les choses de cette façon, on ne peut que se trouver confronté à des paradoxes : celui d'une société qui produit de la richesse tout en l'interdisant; celui aussi d'une Église puissante qui s'enrichit et administre sa fortune tout en la condamnant.

Dans une même perspective, l'intérêt croissant des théologiens du $\mathrm{XIII}^{\mathrm{e}}$ siècle pour les réalités économiques est généralement interprété comme un changement de cap de l'institution ecclésiale face aux mutations de la conjoncture et à l'essor de l'économie marchande. Après une longue phase où la morale ecclésiastique du haut Moyen Âge aurait brimé le développement économique en condamnant notamment les activités commerciales pour leur substituer un modèle d'échanges fondé sur le don/contre-don ${ }^{2}$, l'Église du bas Moyen Âge, dépassée en quelque sorte par l'évolution «naturelle» de la société, aurait choisi de «suivre» le mouvement pour ne pas se trouver dépassée ${ }^{3}$, et de l'encadrer en exerçant un contrôle moral à travers l'interdiction de certaines pratiques comme l'usure ${ }^{4}$. Ces positions, très répandues et largement admises, n'épuisent pas pour autant les approches possibles des rapports entre économie et religion. Surtout, leur notoriété ne doit pas nous dispenser de les soumettre à une analyse historiographique ${ }^{5}$, car, au fond, il s'agit du vieux schéma de l'apparition et de l'essor du marché moderne liés à l'émancipation d'une bourgeoisie s'affranchissant peu à peu des interdits de l'Église. Un schéma qui ne fait qu'appliquer à l'histoire médiévale les positions et les conceptions idéologiques du XIXe siècle.

Dans un livre récent, Giacomo Todeschini propose une approche renouvelée des rapports entre l'Église médiévale et le monde des échanges

2. P. Grierson, «Commerce in the Dark Ages. Critique of Evidence», Transactions of the Royal Historical Society, 5e série, 9 (1959), p. 127-139; même s'il peut être critiqué à cause des présupposés évolutionnistes dont il fait preuve, cet article a ancré l'idée que le système du don/contredon est le paradigme anthropologique le plus apte à expliquer les liens socio-économiques au haut Moyen Âge, en limitant ainsi à ce concept l'approche des questions économiques pour l'époque qui précède le $\mathrm{XI}^{\mathrm{e}}$-XII ${ }^{\mathrm{e}}$ siècle (cf. J.-P. DeVROEY, Économie rurale et société dans l'Europe franque. Fondements matériels, échanges, lien social, Paris, 2003, p. 175 et suiv.).

3. Déjà R.H. Tawney, Religion and the Rise of Capitalism, Londres, 1926.

4. Cette opinion semble partagée, cf. entre autres: E. SCHREIBER, Die volkswirtschaftlichen Anschauungen der Scholastik, seit Thomas von Aquin, Iéna, 1913; J.T. Noonan, The Scolastic Analysis of Usury, Cambridge Mass., 1957; R. De Roover, La Pensée économique des Scolastiques, Montréal-Paris, 1971; J. Le GoFf, Marchands et banquiers du Moyen Âge, Paris, 1956; ID., La Bourse et la vie. Économie et religion au Moyen Âge, Paris, 1986.

5. G. Todeschini, Il prezzo della salvezza. Lessici medievali del pensiero economico, Rome, 1994. 
matériels ${ }^{6}$. En France, son œuvre n'est connue qu'indirectement, rares étant les lecteurs de première main de ses nombreux ouvrages et articles. La traduction française de Richesse franciscaine comble en partie cette lacune historiographique francophone et permet d'accéder à l'un des thèmes chers à l'auteur: la réflexion des maîtres franciscains, entre le XIII ${ }^{e}$ et le $\mathrm{XVI}^{\mathrm{e}}$ siècle, sur la pauvreté volontaire et donc aussi sur l'argent, les prix, les contrats, les rentes et les échanges ${ }^{7}$. Le lecteur qui s'intéresse au franciscanisme est entraîné au cœur d'une discussion plus ample dépassant la thématique strictement religieuse et le cadre de l'histoire de l'Ordre, pour atteindre le problème des rapports complexes, et à nos yeux souvent contradictoires, entre l'institution ecclésiale et le monde matériel. Comme il le fait depuis ses premiers travaux, l'auteur propose ainsi, dans cette synthèse au titre provocateur, une méthode pour analyser le discours ecclésiastique médiéval, en tant qu'objet historiquement daté, en réinsérant les catégories de pensée et le langage des sources dans des structures intellectuelles et culturelles spécifiques au monde chrétien du Moyen Âge. Il met ainsi au jour non pas l'existence d'une «pensée» ou d'une «science» économique médiévale, mais la construction de lexiques et de catégories propres à cette société chrétienne et aptes à décrire et à donner forme au réel ${ }^{8}$. Le discours de G. Todeschini sur les franciscains fait partie d'une lecture beaucoup plus large qu'il donne de la théologie médiévale,

6. G. TODESCHINI, Richesse franciscaine. De la pauvreté volontaire à la société de marché, Verdier, 2008 (éd. it., Bologne, 2004).

7. Sur ces thèmes, voir en France les travaux de S. PIron, «L'apparition du resicum en Méditerranée occidentale, $\mathrm{XII}^{\mathrm{e}}$-XIII ${ }^{\mathrm{e}}$ siècles », dans E. Collas-Heddeland, M. CoudRY, O. Kammerer, A.J. Lemaître, B. Martin éd., Pour une histoire culturelle du risque. Genèse, évolution, actualité du concept dans les sociétés occidentales, Strasbourg, 2004, p. 59-76; ID., «Perfection évangélique et moralité civile. Pierre de Jean Olivi et l'éthique économique franciscaine», dans B. Molina, G. SCARCia éd., Ideologia del credito fra Tre e Quattrocento : dall'Astesano ad Angelo da Chivasso. Atti del convegno internazionale (Asti, 2000), Asti, 2001, p. 103-143; ID., «Marchands et confesseurs. Le Traité des contrats d'Olivi dans son contexte (Narbonne, fin XIII'-début XIV ${ }^{\mathrm{e}}$ siècle)», dans L'Argent au Moyen Âge. XXVIII ${ }^{e}$ Congrès de la SHMESP (Clermont-Ferrand, 1997), Paris, 1998, p. 289-308; ID., «Prêts charitables et opérations capitalistes dans l'éthique franciscaine des contrats monétaires », dans L.Fontaine, G. Postel-Vinay, J.-L. Rosenthal et P. Servais éd., Des personnes aux institutions. Réseaux et culture du crédit du XVI au XXe siècle en Europe, Louvain-la-Neuve, 1997, p. 11-27; ID., «Vœu et contrat chez Pierre de Jean Olivi», Cahiers du Centre de recherches historiques, 16 (1996), p. 43-56. En Italie, outre les travaux de G. Todeschini, voir G. CeCCARelli, Il gioco e il peccato. Economia e rischio nel Tardo Medioevo, Bologne, 2003; P. EvANGELISTI, I francescani e la costruzione di uno Stato. Linguaggi politici, valori identitari, progetti di governo in area catalanoaragonese, Padoue, 2006 (Fonti e ricerche, 20).

8. Pour une exposition des principes méthodologiques: G. TodeschinI, «Oeconomica franciscana I. Proposte di una nuova lettura delle fonti dell'etica economica medievale», Rivista di storia e letteratura religiosa, 12 (1976), p. 15-77; ID., «Oeconomica franciscana II. Pietro di Giovanni Olivi come fonte per la storia dell'etica economica medievale», Rivista di storia e letteratura religiosa, 13 (1977), p. 461-494 ; ID., Il prezzo... 
essentiellement entre le $\mathrm{XI}^{\mathrm{e}}$ et le $\mathrm{XVI}^{\mathrm{e}}$ siècle, en remontant souvent aux origines patristiques des lexiques chrétiens dont il fait l'histoire?

Avec la crise actuelle, dont on pourrait attendre qu'elle suscite une critique du système économique mondialisé, le monde s'interroge sur les notions d' «économie morale » et de «moralisation du marché ${ }^{10}$. L'attention grandit pour les croisements entre histoire économique et histoire religieuse. La question des rapports entre la spiritualité des ordres mendiants et l'économie des couvents est le thème central du volume collectif dirigé par Nicole Bériou et Jacques Chiffoleau ${ }^{11}$. Le dernier ouvrage de Jacques Le Goff consacre une grande partie de ses développements - sous la forme d'un «essai d'anthropologie historique »à la façon dont la société médiévale a considéré l'argent ou la richesse matérielle ${ }^{12}$. L'approche de G. Todeschini permet d'engager le débat avec d'autres tendances méthodologiques, du point de vue particulier des positions du franciscanisme sur les échanges, l'administration et la circulation de la richesse.

\section{Une vision franciscaine de la société}

Commençons peut-être par exposer très rapidement le thème principal de Richesse franciscaine avant d'en présenter plus en détail la méthode et les apports historiographiques. G. Todeschini montre comment l'adoption d'un mode de vie évangélique et la réflexion sur la pauvreté volontaire ont conduit les franciscains à un choix quelque peu paradoxal à nos yeux : sans condamner a priori la richesse du siècle, ils se sont intéressés à toutes les expressions de la vie économique et

9. Parmi les très nombreux titres sur les vocabulaires économiques du christianisme, voir notamment: G. TodeschinI, I mercanti e il tempio. La società cristiana e il circolo virtuoso della ricchezza fra Medioevo e Età Moderna, Bologne, 2002, où les thèmes résumés dans Richesse franciscaine trouvent un développement plus ample et complet; voir aussi: ID., «Quantum Valet? Alle origini di un'economia della povertà », Bullettino dell'Istituto Storico Italiano per il Medioevo, 98 (1992), p. 173-234; ID., «I vocabolari dell'analisi economica fra Alto e Basso Medioevo: dai lessici della disciplina monastica ai lessici antiusurari (X-XIII)», Rivista Storica Italiana, 110/3 (1998), p. 781-833; ID., «Carità e profitto nella dottrina economica francescana da Bonaventura all'Olivi », Franciscan Studies, 60 (2002), p. 325-339.

10. Cf. l'engouement pour divers travaux sur ces thèmes: A. SEN, L'Économie est une science morale, Paris, 2003; les nombreux travaux et articles pour le grand public de Joseph Stiglitz (cf. ID., Le Triomphe de la cupidité, Paris, 2010). La parution en France de L. FonTaINE, L'Économie morale. Pauvreté, crédit et confiance dans l'Europe pré-industrielle, Paris, 2008 et d'articles récents : D. Margairaz et P. Minard, «Marché des subsistances et économie morale: ce que "taxer" veut dire», Annales historiques de la Révolution française, 352/2 (2008), p. 53-99; D. FAssin, «Les économies morales revisitées », Annales. Histoire Sciences Sociales, 64 (2009), p. 1237-1266.

11. N. BÉriou et J. Chiffoleau dir., Économie et religion. L'expérience des ordres mendiants $\left(X I I I^{e}-X V^{e}\right)$, Lyon, 2009 (Histoire et archéologie médiévales, 21).

12. J. Le GoFf, Le Moyen Âge et l'argent. Essai d'anthropologie historique, Paris, 2010. 
sociale de leur temps, en analysant les différentes formes de rapport aux biens matériels et au monde. Les maîtres de l'Ordre ont livré à travers le filtre de la pauvreté évangélique une lecture de la société dans laquelle ils vivaient. Si l'on trouve dès les premiers textes franciscains, ceux de François et de Thomas de Celano en particulier, la volonté de réfléchir sur l'état de pauvreté et sur les notions de besoin relatif, de nécessité et d'usage des biens, à partir des années 1230-1240, une véritable production théologique héritière des Pères de l'Église et de la réflexion des clercs réformateurs s'attache à examiner les problèmes de la circulation de l'argent et de la formation des prix, les formes du contrat marchand, les règles de fonctionnement des échanges. Le comportement et le rôle du marchand ainsi que de tous les acteurs des échanges dans la société sont définis et analysés dans le cadre de cette réflexion théologique sur le salut de l'âme et sur le bien-fondé du mode de vie des disciples de saint François. Étroitement lié à ces questions, un autre thème préoccupe les théologiens de l'Ordre : la civitas christiana et la notion de bien commun, de bonheur civique, entendu comme le bon fonctionnement et le bon gouvernement de la res publica ${ }^{13}$. G. Todeschini montre que la réflexion franciscaine sur l'économie - terme qu'il n'entend pas dans le sens moderne de science économique - a produit un modèle de société où les acteurs économiques contribuent au bien de la communauté civique par leur capacité à faire fructifier une richesse privée à travers le commerce. Les écrits des franciscains associent en effet le bien-être collectif à la circulation de cette richesse marchande et désignent comme stérile et contraire au bien commun la richesse thésaurisée du propriétaire foncier et de l'aristocrate.

Le livre aborde aussi les conséquences de cette vision du monde où chacun semble trouver sa place et sa propre valeur en fonction de sa capacité à faire circuler la richesse, par largitio chez le propriétaire foncier et l'aristocrate, par ses qualités et sa maîtrise technique pour le marchand: qu'en est-il en revanche, dans la vision franciscaine de la société, de ceux qui ne contribuent pas activement au bonheur citadin, de ceux qui n'ont pas les moyens ou les connaissances nécessaires pour participer au circuit du commerce utile à la communauté ? Comment faire participer au bien commun les pauvres, les malades, les marginaux ? Au-delà du commerce, quels sont les types d'échanges qui peuvent garantir au pauvre l'insertion dans le tissu social? Quelle est la place des juifs, des usuriers et des infames dans une cité chrétienne qui associe depuis des siècles les notions de bona fama, de fides religieuse, de caritas et de fiabilité économique ? La production des franciscains sur ces thèmes est aussi abordée dans ce livre qui introduit ainsi aux nouvelles recherches menées ces dernières années par G. Todeschini autour des notions de bonum commune, d'infamia facti et plus généralement des mécanismes

13. Sur ces thèmes voir aussi les travaux de P. Evangelisti, I francescani e la costruzione di uno Stato... 
de l'exclusion sociale, des conceptions plaçant le crédit et l'endettement au cœur de la formation de la citoyenneté ${ }^{14}$. G. Todeschini montre comment les maîtres franciscains ont produit et utilisé un lexique grâce auquel la communauté des fidèles en marche vers le salut était décrite, définie et organisée comme une société fondée par des modalités propres d'échanger, de faire circuler les biens matériels et immatériels.

Au moment de refermer ce livre, son titre révèle toute sa justesse. Il pourrait susciter quelque réserve en raison non seulement de l'association de la pauvreté volontaire à la société de marché, mais aussi du recours à ce concept anachronique et à une formule semblant prendre le parti de l'évolution de l'une à l'autre ${ }^{15}$. Toutefois, l'auteur n'y établit pas un lien de causalité, mais donne les deux extrêmes temporels du long parcours lexical qu'il a entrepris d'analyser, dès l'affirmation institutionnelle du mode de vie pauvre, incarné dans la règle franciscaine approuvée par la papauté, au seuil de la modernité. Ce parcours est tout autre chose qu'une évolution simple et linéaire. Histoire des lexiques et des catégories, et non des théories ou de la pensée, il ne saurait être réduit à l'idée simpliste et fausse selon laquelle les franciscains auraient «inventé» l'économie capitaliste, auraient compris les lois de la science économique ou seraient les précurseurs de l'économie classique. Loin d'affirmer un rapport de cause à effet entre la réflexion franciscaine sur l'argent et l'économie de marché moderne, Todeschini fait la démonstration que «ce fut la religiosité la plus rigoureuse celles des adeptes de la pauvreté évangélique - qui élabora une large part du vocabulaire de l'économie occidentale». Le problème ne se pose donc pas en termes d'invention théorique plus ou moins consciente, mais d'élaboration d'un vocabulaire nouveau et de sa stratification dans le temps. Les textes franciscains définissent un langage concret apte à exprimer les nouveaux besoins du monde occidental et ses transformations. Ce lexique, qui mêle réflexion théologique et observation du réel, crée de nouvelles catégories pour penser le monde et la société, pour appréhender la réalité des échanges et de la circulation de la richesse. En définissant les comportements des nouveaux acteurs économiques et en indiquant leur conformité au salut, il impose les nouvelles règles de l'ordre social des cités. Todeschini restitue en somme au langage des maîtres théologiens du franciscanisme son pouvoir de création de la réalité.

Cela dit, il faut insister sur un point: les franciscains n'ont pas théorisé l'économie de marché et ils n'en sont pas les précurseurs. Comme le rappelle

14. Sur ces thèmes, voir: G. TODESCHINI, Visibilmente crudeli. Malviventi, persone sospette e gente qualunque dal Medioevo all'Età moderna, Bologne, 2007 et son tout dernier ouvrage: ID., Come Giuda. La gente qualunque e i giochi dell'economia all'inizio dell'Età moderna, Bologne, à paraître en juin 2011.

15. Voir les critiques de J. Le Goff, Le Moyen Âge et l'argent..., p. 200-201. 
l'introduction du livre, il ne s'agit pas de prendre les franciscains pour des «prédécesseurs d'Adam Smith» découvrant les lois du marché avant la lettre, mais de considérer que leur conception de la pauvreté s'exprime à travers un langage, qui nous apparaît à la fois économique et théologique, «et qu'elle a, de ce fait, formé un certain nombre des catégories fondamentales du raisonnement économique occidental, et notamment celles des protestants. Il ne s'agit donc ni d'une idéalisation de l' "esprit" du capitalisme dans une version catholique, ni d'une anticipation de la naissance de la science économique ${ }^{16}{ }$. La complexité du terrain historiographique, dans lequel s'insèrent de façon critique ses travaux, pousse l'auteur à prendre nettement position, d'autant plus que le malentendu est trop souvent aux aguets ${ }^{17}$. On le comprend, la "société de marché» de G. Todeschini n'est pas celle dans laquelle nous sommes censés vivre. Bien qu'anachronique, le concept sert à décrire une forme de «société de marché» historiquement datée et son ambiguïté chronologique invite précisément le lecteur à prendre conscience des écarts entre celle-ci et la nôtre.

\section{Tendances et certitudes de l'historiographie}

Avant d'entreprendre une quelconque recherche sur les rapports entre économie et religion au Moyen Âge, il est nécessaire de se confronter à l'imposante littérature sur ce que l'on appelle, de manière fort imprécise et anachronique, la «pensée économique médiévale». Le travail de G. Todeschini, qui remet en question l'existence même de ce type de pensée au Moyen Âge, a été accompagné précisément d'un décryptage critique de cette historiographie ${ }^{18}$. Dans Il prezzo della salvezza, l'auteur retrace les origines des tendances contemporaines sur le sujet, en remontant jusqu'au moment où la «pensée économique médiévale» naît comme objet d'étude ${ }^{19}$. Il a pu ainsi montrer que les cadres principaux dans lesquels évoluent les études du $\mathrm{Xx}^{\mathrm{e}}$ siècle sur ce sujet s'enracinent dans la tradition historique «romantico-nationaliste» allemande et française de la deuxième moitié du XIX ${ }^{\mathrm{e}}$ siècle qui donne naissance à cet objet historique ${ }^{20}$. Au cœur de cette littérature abondante et complexe, presque inconnue aujourd'hui,

16. G. TodeschinI, Richesse franciscaine..., p. 10.

17. Cf. J. Le Goff, Le Moyen Âge et l'argent..., p. 202 et 207.

18. Il est regrettable que B. Clavero n'ait pas lu le travail de G. Todeschini avant de le critiquer, cela lui aurait évité de l'associer à une historiographie à laquelle il s'est toujours opposé: cf. B. Clavero, La Grâce du don. Anthropologie catholique de l'économie moderne, Paris, 1996, p. 20 et n. 29, où par exemple G. Todeschini est étrangement associé à deux auteurs qu'il critique abondamment, L.K. Little et A. Spicciani.

19. Pour une reconstruction du développement des études sur la «pensée économique» du milieu du XIXe siècle aux années 1990, voir G. ToDEsChInI, Il prezzo..., p. 39-113.

20. Ibid., p. 39. 
germent la plupart des idées qui caractérisent encore de nos jours les opinions des historiens sur les rapports entre l'économie et la religion.

Dans le premier tiers du $\mathrm{xx}^{\mathrm{e}}$ siècle, les historiens qui traitent des «conceptions économiques» du Moyen Âge ont déjà à leur disposition plusieurs théories et hypothèses de travail sur le sujet. D'un côté, le modèle historiographique de Werner Sombart, qui relie les origines du «capitalisme» aux pratiques économiques juives, en reléguant la chrétienté médiévale à la seule sphère agricole et artisanale et à l'ignorance des lois économiques jusqu' au $\mathrm{Xv}^{\mathrm{e}}$ siècle $^{21}$. À la vision de W. Sombart s'oppose l'hypothèse de Max Weber, qui propose plutôt de souligner les affinités entre l'éthique religieuse protestante et $l^{\prime}$ ' esprit du capitalisme ${ }^{22}$ ». À côté de ces deux positions, le catholique Ignaz Seipel et surtout le protestant Ernest Troeltsch promeuvent, au début du siècle, une conception faisant remonter au christianisme patristique les origines d'une organisation sociale rationnelle, attentive à l'équilibre entre profits individuels et justice sociale ${ }^{23}$. Ces deux auteurs décrivent une sorte de conciliation progressive entre la morale chrétienne et la société matérielle, en direction d'une alliance, voire d'un compromis entre économie et christianisme avec pour objectif le gouvernement nécessaire du monde terrestre. L'intention de fond est de découvrir comment et quand le message évangélique a pu conduire à la définition théorique de la société capitaliste. En particulier, les adversaires de l'hypothèse de W. Sombart construisent l'image d'un bas Moyen Âge chrétien capable de produire une pensée économique en phase avec le développement et le progrès de ce type de société.

Cette position remplace certaines idées de l'historiographie catholique de la fin du XIX ${ }^{e}$ siècle, selon lesquelles la chrétienté, à partir de l'époque patristique, forte d'une morale économique très ancienne, aurait construit son identité médiévale sur l'aversion pure et simple pour la richesse et le rejet des biens matériels ${ }^{24}$. Dans le premier tiers du $\mathrm{Xx}^{\mathrm{e}}$ siècle, cette idée est relue plutôt en termes de décalage entre une théorie économique chrétienne plongeant ses racines

21. W. Sombart, Die Juden un das Wirtschaftsleben, Leipzig, 1913; voir pour plus de détails la bibliographie indiquée par G. Todeschini, Il prezzo..., p. 40.

22. M. WeBER, L'Éthique protestante et l'esprit du capitalisme, Paris, 2000 ( $1^{\text {re }}$ éd. 1904).

23. E. Troeltsch, Die Soziallehren...; I. SeIPEL, Die wirtschaftlichen Lehren der Kirchenväter, Vienne, 1907. L'influence des travaux de E. Troeltsch est immédiate surtout dans les milieux allemand et anglo-saxon, puis italien, tandis qu'en France il faudra attendre les années 1960: cf. J. SÉGUY, «Introduction. Ernst Troeltsch et ses Soziallehren», Archives des sciences sociales des religions, 11 (1961), p. 7-14.

24. Cf. F.X. Funk, Geschichte des kirchlichen Zinsverboten, Tübingen, 1876; H. ConTZEN, Geschichte der volkswirtschaftlichen Literatur des Mittelalters, Berlin, 1869; V. BRANTs, L'Économie politique au Moyen Âge: esquisse des théories économiques professées par les écrivains des XII et XIV siècles, Louvain, 1895. 
dans l'Antiquité et le développement inévitable d'une logique de marché. On tente donc non seulement d'apporter des justifications historiques à une volonté de conciliation, propre notamment aux positions du christianisme social, de la pensée chrétienne avec le monde contemporain ${ }^{25}$; mais, plus important encore, est élaborée l'idée que la modernité économique de l'Occident s'enracine dans le Moyen Âge et l'on commence à situer les véritables débuts de l'aventure économique européenne au cœur de la pensée théologico-morale des scolastiques. Sous l'influence des thèses de $\mathrm{M}$. Weber sur le protestantisme, on commence à se demander si la réflexion économique des Scolastiques, en tant que manifestation de l'éthique sociale chrétienne, n'a pas favorisé la croissance médiévale et le passage du féodalisme à l'économie mercantile. L'idée que la doctrine chrétienne ne fait qu'enregistrer, malgré elle, l'évolution naturelle de l'économie laisse la place à la conviction que la «pensée économique» médiévale appartient intimement à l'éthique sociale de l'Église, dont elle serait l'un des aspects majeurs, et qu'elle opère au sein de la société dans le sens d'un contrôle moral de pratiques économiques naturellement injustes ${ }^{26}$. La difficulté méthodologique à rendre compte des conceptions chrétiennes médiévales sera donc éliminée par les nouvelles positions de l'historiographie du début du $\mathrm{xx}^{\mathrm{e}}$ siècle, pour qui la chrétienté, et tout particulièrement le thomisme, a été capable de produire une réflexion économique du fait même de son éthique rationalisatrice, tournée vers le gouvernement du monde.

Il est impossible de rendre compte ici de manière satisfaisante de toutes les positions qui apparaissent à partir des années 1930, leur éventail devenant de plus en plus large et difficile à résumer. Un nouveau travail de déchiffrage et de critique historiographique se révèle nécessaire aujourd'hui, pour explorer les méandres de cette littérature et examiner l'état contemporain de la question ${ }^{27}$. Limitons-nous ici à examiner avec $\mathrm{G}$. Todeschini les principaux griefs qu'il adresse aux historiens de la «pensée économique» et essayons de saisir les principaux apports méthodologiques de ses travaux.

La plupart des travaux sont consacrés à une reconstitution positiviste du discours chrétien sur la richesse dont le véritable essor est fixé autour du $\mathrm{XII}^{\mathrm{e}}$ siècle, ce qui coïnciderait avec une phase de développement économique et d'ascension d'une bourgeoisie marchande. Le choix des sources a été conditionné

25. Pour ces questions, voir V. Toneatto, Marchands et banquiers du Seigneur, Rennes, à paraitre en 2011.

26. Cf. I. SEIPEL, Die wirtschaftlichen Lehren...

27. Je me permets d'annoncer un premier point sur la question dans ma thèse (Marchands et banquiers du Seigneur, à paraître) et un travail en préparation ayant pour objectif de présenter un bilan historiographique sur l'étude des rapports entre économie et religion au Moyen Âge, l'une des dernières tentatives étant celle B. Clavero, La Grâce du don... 
par une perspective sectorielle: J. Le Goff recherche les positions théologicomorales dans les sermons ${ }^{28}$; l'émergence de théories économiques est retracée dans la littérature quodlibétique, les quaestiones des écoles médiévales et les summae théologiques; ou alors on s'attache uniquement aux définitions juridiques et aux interdits canoniques ${ }^{29}$. Les historiens ont identifié une série d'auteurs, Thomas d'Aquin, Jean Duns Scot, Nicolas Oresme, Bernardin de Sienne, à qui ils attribuent la découverte de certaines lois économiques, voire des fondements de la science économique ${ }^{30}$. Sur la base d'un groupe assez restreint d'auteurs et de sources, ont été analysés des sujets bien délimités : les interdits sur la monnaie ou sur l'usure, identifiés comme le fond de la théorie économique médiévale ou d'un rapport spécifique à la richesse ${ }^{31}$.

Cette approche présuppose que les lois économiques existent en tant qu'invariants historiques avant même leur théorisation par Adam Smith et les économistes du $\mathrm{XIX}^{\mathrm{e}}$ siècle. L'histoire classique de la pensée économique médiévale prend ainsi comme horizon de l'enquête non pas le Moyen Âge, mais l'économie de marché moderne, considérée comme la forme «normale» et pleinement aboutie de l'économie par excellence, dont il faut rechercher les racines. Les analyses sont souvent orientées vers la recherche des précédents des doctrines contemporaines ou des obstacles à leur épanouissement, plutôt que vers la compréhension des modalités proprement médiévales de penser et de pratiquer les activités relevant du quotidien matériel, des échanges, de la production et de la circulation des biens ${ }^{32}$.

L'habitude d'isoler un certain nombre d'autorités, de thèmes, de sources dans la masse des textes médiévaux - en s'occupant seulement d'œuvres qui affrontent des thèmes explicitement chrématistiques (appliqués à des réalités politiques, selon le modèle aristotélicien) - conduit aussi à ignorer toutes les sources qui parlent de comportements liés à l'administration de la richesse dans des contextes généralement éthico-spirituels et normatifs - comme par

28. Cf. à propos de l'interdiction de l'usure dans la prédication: J. LE GoFf, «The Usurer and Purgatory », dans The Dawn of Modern Banking, New Haven-Londres, 1979, p. 25-53 ; ID., La Bourse et la vie...

29. G. TodeschinI, Il prezzo..., p. 18-19 et de manière plus approfondie p. 29-35.

30. Il s'agit de la position classique de J. SchumPETER, History of Economic Analysis, New-York, 1954; A. SAPORI, Studi di storia economica, 2 t., Florence, 1955-1967; R. DE RoOver, La Pensée économique...; O. Langholm, Price and Value in the Aristotelian Tradition. A Study in Scholastic Economic sources, Oslo, 1979; ID., Wealth and Money in the Aristotelian Tradition. A Study in Scholastic Economic sources, Oslo, 1983, ID., Economics in Medieval Schools : Wealth, Exchange, Value according to the Paris Theological Tradition, Leyde, 1992.

31. J.T. Noonan, The Scolastic Analysis...; O. Langholm, The Aristotelian Analysis of Usury, Oslo, 1984; et les travaux de J. Le GoFf.

32. Il s'agit de l'une des critiques fondamentales faites à l'historiographie sur la «pensée économique» : G. TodeschinI, Il prezzo..., p. 29 passim. 
exemple les règles des moines et des Ordres religieux ou les canons conciliaires. L'intérêt pour le bas Moyen Âge, où l'on cherche à tort la transition entre les économies du don/contre-don et du marché, implique de négliger presque toute la production ecclésiastique médiévale entre le $\mathrm{v}^{\mathrm{e}}$ et le $\mathrm{xl}^{\mathrm{e}}$ siècle ainsi que le rôle majeur des réformateurs dans le développement et la transformation des constructions discursives postérieures ${ }^{33}$.

Lorsque cette reconstruction d'improbables théories économiques médiévales pré-capitalistes est jugée à juste titre anachronique et téléologique car il est impossible d'affirmer l'existence au Moyen Âge de l' «économie» en tant que science théorique -, le discours de l'Église est néanmoins perçu comme un obstacle ou un frein au développement de l'économie laïque ${ }^{34}$. Même lorsque l'on affirme à raison que la science économique n'existe pas au Moyen Âge, l'économie, elle, est encore considérée comme une réalité objective, laïque, existant indépendamment des institutions médiévales et surtout de l'Église, et s'exprimant à travers un ensemble de pratiques et de mécanismes propres, quasi naturels, n'attendant qu'une chose ou presque: que la bourgeoisie s'émancipe des contraintes imposées par l'Église et se mette à théoriser et à rationaliser ces pratiques pour son propre compte. En attendant, cette réalité se heurterait à un système conceptuel, celui de la théologie et de la morale chrétienne, qui lui serait opposé depuis l'Antiquité et qui s'exprimerait avant tout par des interdits (l'usure) et par des exhortations au bon comportement économique. L'idée est donc ancienne et rejoint, au niveau des présupposés théoriques, les travaux qui voient au contraire dans les réflexions scolastiques l'annonce de la modernité et du progrès: le monde de l'économie appartiendrait naturellement aux laïcs et l'Église ferait incursion de manière plus ou moins justifiée et utile dans un domaine qui lui est foncièrement étranger. De ce point de vue, l'Église médiévale en tant que structure externe aux pratiques économiques ne peut que constituer un frein ou une sollicitation, selon les positions des historiens ${ }^{35}$.

33. Ibid., p. 32 et p. 101 passim.

34. G. Todeschini (Il prezzo..., p. 15 et n. 7) cite comme exemple J. LE Goff, La Bourse et la vie...

35. Selon J. Schumpeter (History of Economic Analysis...), la morale ecclésiastique a toujours freiné le développement de l'économie et seulement grâce à l'influence d'Aristote et à l'essor économique de la fin du Moyen Âge, elle a pu découvrir les fondements de la science économique. Selon d'autres auteurs, la résistance envers les logiques marchandes aurait eu pour conséquence d'aiguiser les esprits en favorisant la recherche de nouvelles techniques financières et commerciales de la part des marchands, telles que la lettre de change. Cette position présuppose une relation conflictuelle mais fructueuse entre éthique chrétienne et monde des marchands débouchant sur l'émancipation de ce dernier. Elle se retrouve surtout dans le milieu historiographique anglo-americain et français: R. De Roover, La Pensée économique...; Y. Renouard, Les Hommes d'affaires italiens au Moyen Âge, Paris, 1949; J.T. Noonan, The Scholastic Analysis...; J. Le GoFf, Marchands et banquiers. 
Pour affirmer que l'économie n'existe pas au Moyen Âge en tant que domaine structurellement indépendant par rapport aux autres aspects de la vie des hommes en société, l'historiographie s'est appuyée sur K. Polanyi ${ }^{36}$. Selon une perspective anthropologique, en effet, dans une société pré-moderne, la sphère des échanges et de la production de biens ne peut être séparée du domaine de la religion et des rapports sociaux mais elle est «enchâssée» dans la société ellemême ${ }^{37}$. L'application de cette vision des choses au Moyen Âge porte à penser que l'économie n'a justement pas pu se construire comme sphère autonome car les interdictions ecclésiastiques n'ont pas permis le développement d'une activité économique. La condamnation de l'avarice et de l'usure, le refus de l'enrichissement, l'appel à une économie du don seraient donc parmi les causes immédiates du sous-développement de la société médiévale. Ces présupposés infléchissent la vision d'une société médiévale appelée à se développer, mais maintenue à un stade primitif à cause du frein imposé par l'Église. Pour Todeschini, affirmer que l'économie moderne n'existe pas au Moyen Âge à cause de (ou grâce à) la condamnation de l'usure et de l'avarice ou l'impératif de caritas ne suffit pas à rendre compte des formes d'organisation sociale et de rapport à la richesse, en l'absence d'une analyse serrée des pratiques discursives ecclésiastiques pour saisir le sens et la portée de ces interdictions.

\section{Du cloisonnement à l'ouverture : les lexiques économiques}

Un tournant historiographique se situe dans les années 1950-1960, lorsque Ovidio Capitani propose d'abandonner l'idée d'une théorie économique du bas Moyen Âge ${ }^{38}$. O. Capitani souhaite rechercher dans les sources scolastiques la présence d'un discours stratifié et structuré selon des orientations diverses, plutôt qu'une théorie à part entière synthétisant de manière cohérente la pratique économique ${ }^{39}$. Il privilégie l'attention au vocabulaire, se tournant vers la construction d'un lexique propre à la période médiévale, sans plus attribuer aux scolastiques la découverte de lois économiques.

36. C'est encore le cas de J. Le Goff, Le Moyen Âge et l'argent.

37. K. Polanyı, La Grande Transformation. Aux origines politiques et économiques de notre temps, Paris, 1983.

38. Voir par exemple O. CAPITANI, «La venditio ad terminum nella valutazione morale di S. Tommaso e di Remigio de'Girolami », Bullettino dell'Istituto Storico Italiano per il Medioevo, 70 (1958), p. 299-363 ; ID., «Il De peccato usure di Remigio de'Girolami», Studi Medievali, $3^{\text {e }}$ sér., 6 (1965), p. 537-661.

39. Cf. pour la bibliographie G. TodeschinI, Il prezzo..., p. 78-79. Cf. entre autres: O. CAPITANI éd., L'etica economica medievale, Bologne, 1974; ID. éd., Una economia politica nel Medioevo, Bologne, 1987. 
Il ne s'agit plus, dorénavant, d'analyser le système théorique de la pensée médiévale, mais d'étudier la formation d'un langage «éthico-économique ${ }^{40}$ ». Ce sera la tâche entreprise par G. Todeschini au milieu des années 1970, grâce à un projet à long terme de relecture et de réinterprétation des sources médiévales. Écartant comme objet d'étude une «pensée» économique chrétienne faussement unitaire ou les «théories» des scolastiques et déjouant surtout l'opposition entre religion et économie, G. Todeschini se tourne plutôt vers l'analyse des vocabulaires éthico-économiques chrétiens qui se forment dès la fin de l'Antiquité et se stratifient tout au long du Moyen Âge dans les cadres théologiques et normatifs de la littérature épiscopale et monastique. En évitant de collectionner les auteurs dits représentatifs et sans se borner à la seule analyse des sources scolastiques abordant explicitement des thèmes économiques, il envisage un travail de longue haleine sur une production textuelle aux multiples visages, qui va des sermons et des traités théologiques et exégétiques à la littérature normative canonique et pénitentielle, du $\mathrm{v}^{\mathrm{e}}$ au $\mathrm{Xv}^{\mathrm{e}}$ siècle $^{41}$. À l'intérieur de ce système textuel complexe caractérisé par des bases bibliques et patristiques, Todeschini analyse les expressions et les catégories que les ecclésiastiques emploient pour parler de la réalité changeante du quotidien matériel.La vie des mots ne pouvant être saisie si l'on se limite à travailler sur un seul auteur ou une seule catégorie de sources, il est nécessaire de considérer une littérature volumineuse, dans l'espace et le temps, pour vérifier les strates chronologiques de formation du sens des mots, les influences contemporaines d'un texte à l'autre et les adaptations à des contextes divers. Cette démarche rend caduque toute tentative d'écarter certaines œuvres sous prétexte qu'elles seraient marginales, peu scientifiques ou non représentatives ${ }^{42}$.

Cet intérêt pour les mots naît de l'exigence de comprendre la signification des emplois médiévaux de certains termes - avaritia, usura, caritas, administratio - qui sont trop souvent interprétés à travers le filtre du sens qu'on leur attribue aujourd'hui. Il s'agit de déchiffrer le système médiéval de dénotation dans le domaine «économique». Il s'agit de comprendre les concepts et les conduites

40. Ce terme a été proposé par G. Todeschini, Il prezzo..., p. 119 passim.

41. Après quelques articles consacrés à la période des $\mathrm{V}^{\mathrm{e}}-\mathrm{XI}^{\mathrm{e}}$ siècles, $\mathrm{G}$. Todeschini se concentre surtout sur les derniers siècles du Moyen Âge, tout en conservant dans la structure de ses livres une perspective de longue durée.

42. Cf. à ce sujet la diatribe sur l'utilisation des œuvres de Pierre de Jean Olivi dans : O. CAPITANI éd., Una economia politica... Todeschini a été critiqué par J. KIRSHNER et K. Lo PrETE, «Peter John's Olivi Treatise on Contracts of Sale, Usury and Restitutions : Minorite Economics or Minor Works?», Quaderni fiorentini, 13 (1984), p. 233-286 et par J. LE GoFf, Le Moyen Âge et l'argent..., pour avoir donné trop d'importance à Olivi, alors que sa démarche consiste précisément à vérifier le traitement des thèmes «éthico-économiques» chez une très grande variété d'auteurs et de sources et sur la longue durée. 
qui se cachent derrière un lexique dont le sens peut apparaître tout à fait ordinaire au lecteur moderne. Ce sens qui nous semble évident aujourd'hui est issu, au contraire, d'une stratification historique dont il faut saisir les étapes. Les mots apparaissent comme autant de vestiges mis en contexte et en perspective, comme s'ils étaient restitués dans une stratigraphie. Cette recherche presque archéologique, minutieuse et d'une très grande érudition, des lexiques et des catégories peut alors servir de base à une lecture véritablement anthropologique du discours théologique médiéval, à laquelle se livre l'auteur, pour mieux comprendre l'économie de l'institution ecclésiale.

Procédant de la sorte, G. Todeschini recherche non pas les racines de notre économie moderne, mais l'existence d'une façon propre à la société médiévale d'exprimer la nature des échanges matériels à travers le lien qu'elle établit entre l'ici-bas et l'au-delà. Il montre, par exemple, que, dans les discours des scolastiques, tout ce qui concerne le déroulement des échanges est lié inextricablement à la question du salut et, en même temps, tout ce qui concerne le salut est lié à la façon d'échanger les choses, de se les approprier, de les accumuler ou de les faire circuler ${ }^{43}$. Cette double connotation du langage ecclésiastique remonte à l'époque patristique, au moment où la langue du christianisme met au point des outils de réflexion sur la réalité économique, sociale et politique, à partir d'une perspective théologico-normative qui fixe d'ici-bas les conditions du salut de l'homme ${ }^{44}$. À partir du III ${ }^{\mathrm{e}}$ siècle, les Pères commencent à employer un vocabulaire conceptuel et métaphorique caractérisé par une polyvalence sémantique à la lisière entre matériel et spirituel et se référant à des contextes à la fois transcendants et triviaux ${ }^{45}$. Ils utilisent couramment des métaphores issues du monde économique et commercial gréco-romain pour connoter les nouveaux domaines sémantiques du christianisme, tels que le salut de l'âme ou le rachat des péchés ${ }^{46}$. Ils décrivent ainsi les mécanismes du sacré en termes économiques à travers un discours qui analyse, en même temps, les phénomènes quotidiens et

43. Cf. G. Todeschini, I mercanti e il tempio...

44. Cf. G. TodeschinI, «Quantum valet?... ». Sur les lexiques théologico-économiques entre Antiquité tardive et haut Moyen Âge, je me permets de renvoyer à V. ToneatTo, Marchands et banquiers, à paraître; EAD., «I linguaggi della ricchezza nella testualità omiletica e monastica dal III al IV secolo», dans V. TONEATTO, P. ČERnič et S. PAulitTi éd., Economia monastica. Dalla disciplina del desiderio all'amministrazione razionale, Spolète-Florence, 2004, p. 1-88.

45. C. Mohrmann, «Le latin, langue de la chrétienté occidentale», dans EAD., Études sur le latin des chrétiens, t. I, Rome, 1958, p. 51-81.

46. En particulier sur la métaphore du commerce voir: M. Herz, Sacrum Commercium. Eine begriffsgeschichtliche Studie zur Theologie der Römischen Liturgiesprache, Munich, 1958; R. BOgaert, «Changeur et banquiers chez les Pères de l'Église», Ancient Society, 4 (1973), p. 239270 ; P. RAdici ColACE, «Moneta, linguaggio e pensiero nei Padri della Chiesa fra tradizione pagana e esegesi biblica», Koinonia, 14 (1990), p. 47-64. 
définit le bon comportement économique du fidèle ${ }^{47}$. De cette manière, depuis la fin de l'Antiquité, le discours ecclésiastique associe le transcendant et le sacré à la logique des échanges matériels. Là où les mots de l'économie renvoient à des éléments divins et célestes - dans les métaphores de l'Incarnation comme échange sacré, dans les métaphores monétaires du salut qui offrent aux fidèles des catégories pour penser les mystères de la foi en les visualisant en termes économiques - se trouve le début d'un long parcours de définition lexicale de la réalité sociale, politique, matérielle et spirituelle du chrétien qui se déroule tout au long du Moyen Âge. Les franciscains voient dans le renoncement à la propriété et l'usage modéré les éléments pour comprendre la valeur de l'échange et de la monnaie, pour faire son salut en échangeant les biens matériels, noyau central des pratiques marchandes médiévales. C'est donc au sein d'un vocabulaire chrétien se transformant sur la longue durée que G. Todeschini peut retrouver les logiques lexicales à l'œuvre au Moyen Âge dans la définition de la société, de son fonctionnement matériel et de son rapport particulier au sacré.

En France, des entreprises récentes tentent d'explorer les rapports entre économie et religion au Moyen Âge. C'est le cas tout d'abord de recherches menées à l'université Lyon 2 depuis le début des années $2000^{48}$. Dans la droite ligne des travaux initiés par J. Chiffoleau sur «l'économie de l'institution ecclésiale ${ }^{49}$ », une partie des activités du CIHAM (UMR 5648) s'est concentrée sur l'économie des ordres mendiants à la fin du Moyen Âge, en donnant lieu à la publication d'un volume collectif et à la soutenance d'une thèse de doctorat portant

47. G. Todeschini, «Quantum Valet?», p. 186-187.

48. Cf. par ailleurs, et plus récemment, D. IoGNA PRAT, «Préparer l'au-delà, gérer l'ici-bas : les élites ecclésiastiques, la richesse et l'économie du christianisme (perspectives de travail)», dans J.-P. Devroey, L. Feller et R. Le Jan éd., Les Élites..., p. 59-70. Le thème «économie et religion » fera partie des nouveaux axes de recherche du CEPAM de Nice (UMR 6130): M. LAUwERs éd., $L a$ Dîme au Moyen Âge. Histoire d'un prélèvement ecclésiastique (Collection d'études médiévales de Nice, 12), à paraître; cf. aussi la thèse de E. BAIN, Les Fondements bibliques du discours ecclésiastique sur les riches et pauvres aux XII et XIIt siècles, dir. M. Lauwers.

49. Cf. entre autres: J. Chiffoleau, La Comptabilité de l'au-delà. Les hommes, la mort et la religion dans la région d'Avignon à la fin du Moyen Âge (vers 1320-vers 1480), Rome, 1980 (CEFR, 47); ID., «Pour une économie de l'institution ecclésiale à la fin du Moyen Âge», MEFRM, 96-1 (1984), p. 247-279; ID., «Usus pauper? Les franciscains, la règle et l'argent en Avignon (vers 1360vers 1430)», dans Horizons marins, itinéraires spirituels ( $V^{e}-X V I I I^{e}$ siècles), t. I, Paris, 1987, p. 135149 ; ID., «Les transformations de l'économie paroissiale en Provence (vers 1260-vers 1480)», dans A. PARAVicini Bagliani et V. PAsche éd., La parrochia nel Medioevo : economia, scambi, solidarietà, Actes du colloque de Lausanne (octobre 1991), Rome, 1995, p. 61-117; ID., «Les couvents, l'échange, la religion », conclusions à L'economia dei conventi dei frati minori e predicatori fino alla metà del 1300, Atti del XXXI Convegno internazionale di studi francescani (Assisi, octobre 2003), Spolète, 2004, p. 403-448. 
sur la comptabilité du couvent franciscain d'Avignon ${ }^{50}$. Économie et religion, dirigé par Nicole Bériou et Jacques Chiffoleau, est le fruit d'une entreprise qui a rassemblé vingt-trois chercheurs français et étrangers, à l'occasion de plusieurs journées d'études entre 2002 et 2005 . Une partie de l'équipe a participé activement à l'organisation du trente et unième colloque international d'Assise portant sur l'économie des couvents des mineurs et des prêcheurs jusqu'au milieu du XIV ${ }^{e}$ siècle (2003). Avec le livre de 2009, l'équipe offre un panorama plus vaste du problème par rapport aux horizons touchés par ce colloque: en allant jusqu'à la fin du $\mathrm{Xv}^{\mathrm{e}}$ siècle, les différentes contributions élargissent le champ de recherche, alors que la majorité des travaux, notamment sur les franciscains, avait tendance à s'arrêter à la première moitié du XIV ${ }^{\mathrm{e}}$ siècle et à se focaliser sur les débats autour de la pauvreté. Le questionnaire est distribué en cinq thèmes: les sources du quotidien, la typologie des biens et des revenus, les modalités d'acquisition des biens, les modalités des échanges, le rapport des frères aux biens et à l'argent. Une attention particulière est consacrée à la réflexion sur les sources et au rapport entre les normes et les pratiques. Les études rassemblées dans ce volume se veulent donc attentives aux écrits pragmatiques, aux pratiques quotidiennes des frères et à l'économie concrète des couvents. Elles débouchent aussi, comme le montre bien J. Chiffoleau dans sa conclusion, sur l'étude des rapports à l'au-delà et sur l'histoire de la croyance ${ }^{51}$. La voie semble ainsi ouverte au dialogue et à l'échange avec les travaux de G. Todeschini, qui explorent plus précisément les aspects lexicaux et conceptuels du rapport des franciscains à la richesse et à son utilisation dans le monde, dans le but de mieux comprendre la nature et les caractéristiques propres à l'économie de l'institution ecclésiale ainsi qu'à toutes les pratiques pouvant relever de ce que nous appelons aujourd'hui «économie ${ }^{52}$ ».

Valentina Toneatto - Peking University, Department of History, Beijing 100871, China.

50. C. Lenoble, L'Exercice de la pauvreté. Les franciscains d'Avignon, l'incertitude économique et la circulation de la richesse à la fin du Moyen Âge, thèse, dir. J. Chiffoleau, université Lyon 2, 2010 ; N. BÉrIOU et J. CHIFFOLEAU dir., Économie et religion.

51. J. Chiffoleau, «Conclusion», ibid., p. 707-754.

52. C'est précisément l'une des nouveautés méthodologiques proposées dans la thèse de C. Lenoble, L'Exercice de la pauvreté... Cf. aussi ID., «Dons, rentes, pension et propriété chez les Frères mineurs: un contrat de conversion avignonnais (1368)», dans L. FeLLER éd., Calculs et rationalités dans la seigneurie médiévale : les conversions de redevances entre $\mathrm{XI}^{e}$ et $\mathrm{XV}^{e}$ siècles, Paris, 2009, p. 167-217. 